\title{
Examination results
}

\section{MaRCPsych Part I - Autumn 1995}

Out of a total of 375 candidates who sat the examination, 175 passed.

Esther Maria Sophie Abeln; Maysan AlNufoury; Samuel Ayodele Aladerun; Regi Tharlan Alexander; Spilios Argyropoulos: Narayan Raj Aryal; Parthasarathi Banerjea; Caryl Wynne Barnes; Leonard Ben Bassey; Angela Marian Beardsmore; Elizabeth Beber; Volodia Bezulowsky; Archana Bill; Christina Joy Aderonke Browne; Amanda Marla Burke; Alisdhair James Caldwell; Michael Fintan Stephen Campbell; Jonathan Edmund Campion; Catherine Mary Denise Canavan; Claudiu Dragos Carmaciu; Sarah Deborah Cassar; Grace Chan Yuen Ching; Cheng Kin Shing; Ansar Ali Choudry; Andrew Rupert Cohen; Katherine Ann Cottrell; Janette Sarah Cribb; Susan Nicola Crimlisk; Richard Michael Cullen; Karim Zafar Dar; Malcolm Patrick Darley; Simon Paul Darvill; Sudipto Malay Das; Richard Kenneth Day; Tracey Louise Dibble; Andrew Gavin McIntosh Elmslie; David Edward Fearnley; George Christopher Fox; Jonathan Andrew Fry; Philip Kevin Gothard; Rajvinder Grewal; Joseph Michel Mirhom Guirguis; Alexandra Clare Gundry; Yvonne Woon Ming Ha; Mohamed Salih Hajamodhideen; Rachel Mary Hammill; Elizabeth Helen Hare; Rachel Frances Harland; Kim Maria Frances Hawley; Nicola Patricia Hodelet; Guy John Temple Holloway; Andrew David Holwell; Paul Lindsay Hopper; Beatrice Huang Yun Pui; Susanne Margaret Hume; Princewill Ugochukwu Ikwuagwu; Wai Lan Imrle; Flona Karen Jaffray; Maria Auxiliadora Javaloyes Sanchis; Miles Facundo Jefferson; Benjamin Johnson; Timothy Graeme Johnston; Hugh Jones; Elizabeth Julia Jupp; Elizabeth Morag Macdonald Kennedy; Juliette Mary Kennedy; Eamonn Patrick Kenny; Siobhan Margaret King; Lucie Helena Klenka; Christos Kouimtsidis; Gina Rosalind Kuperberg; Olawale Oladimeji Lagundoye; Kristin Sarah Lancefield; Peter David Le Fevre; Maire Maeve Leonard; Sean Feargal Leonard; Marc Timothy Lester; Elizabeth Johanna Lewis; Fionnuala Mary Lynch; Rosanne Macgregor-Morris; Iain Mackenzie Mackay; Flona Claire Anne Mactier; Sarah Majid; Rakesh Malik; Winnifred Brigid Manning; Matthew James Marchant; Mangesh Marudkar; Marek Marzanski; Philip Richard Matthews; Seonaid Margaret McCallum; Jenny Mackie McCleery; Colm Denis Patrick McDonald; Joy Elizabeth McMahon; Thomas Michael Mario
McMonagle; Andrew James John McNeil; Andrew John Francis Williams Metters; Colin Roy Milliken; David Clive Milnes; Alexander Joel Mitchell; Bruce Jonathan Moore; Maeve Rosemary Moran; Caryl Nell Morgan; Flona Soutar Morrison; Deborah Ann Mountain: Michelle Maria Mulcahy; Ashraf Abdel Raouf Abdel Kader Nasr; Besim Evren Nereli; Graham James Ness; Siobhan Netherwood; Siobhan Ni Bhriain; Matthew Dominic Noble; Mary A O'Brien; Edmond Patrick O'Mahony: Mary Judith O'Neill; Morenike Olufunlola Odebiyi; Muhammed Farouk Okhai; David Philip John Osborn; Julie Owens; Apsara Panikkar; John Pankdw; Stephen Pearce; Nigel Ralph Pearson; Ting Keung Poon; John Antony Powell: Kalpana Prasad; Natalie Mary Pyszora; Sherifal Oyepeju Raji; Ursula Reckermann-Graham; Thomas Joseph Reynolds; Barbora Richardson; Sophie Amanda Roberts; Carmel Jessica Sarah Robinson; Cesar Rodriguez Castello; Belinda Rosalind Routh; Mark Ivan Ainslie Scheepers; Michael Peter Scully; Rajen Shah; John Guy Sharpley; Elita Smiley: Stephanie Marguerite Sommers; Elayathamby Sreedaran; Alison Jane Stansfield; Nicholas Stefanis; Angela Felicity Stevens; Neil Grant Stewart; Christine Annette Hastings Sutherland; Geraldine Mary Swift; Sylvia Tang Sip Shiong; Mary Honor Taylor: Megan Tara Theodoulou; Sally Christina Thomas; Michelle Mary Thrower; Henry Michael Ticehurst; Julie Suzanne Tooth; Tongeji Tungaraza; Therese Aldegona Maria Josephina van Amelsvoort; Mark Damian Vettraino; Robert Alan Walker; Roger Daniel Walters; Neville Alan Warnke; Michael McDonald Warwick; Stuart Watson; Peter Mark Webster; David Antony White; Graeme Edward Whitfield; Koratota Liyanage Wickramarathna; Stephen Edward Wilkinson; Justin Hereward Gwilym Williams; Charlotte Frances Wilson Jones; Hester Clare Womersley; Mark Woodgate; Pamela Ruth Worthington; Yim Heung Wah; Mohammed Radha Salih Bakir Zaini; Mir Moin Zoha.

\section{MRCPsych Part II - Autumn 1995}

Out of a total of 312 candidates who sat the examination, 154 passed.

Madhushree Acharya Goswami; Konstantinos Agathokleous; Andrew Aziz Al-Adwani; Catherine Anne Andrews; Farrah Suhail Anwar; Olakunle Adebisi Ashaye; Jennifer Dawn Ashmead; Suma Balagopal; Dave Alexander Barker; Stephen 
Stuart Barlow; Martin Robert Alexander Bassett: Simon Beer; Jan Beydals; James Gerard Francis Bolton; Owen Matthew Box; Timothy Branton; Ruth Cordelia Garnet Briel; Julia Ann Mary Butler; Peter Robert Byrne; Ftvos Cacoullis; Rebecca Lucy Carleton: Brendan Patrick Jude Cassidy: Kathy Chan Po Man; Murray John Chapman; Chen Teck Meng; Chow Lok Yee; Robert Anthony Clafferty: Mary Nicola Clarke; Xavier Coll I Corominas; Frances Elizabeth Albinia Connell; Claire Anne Craven; Denise Gail Critchlow; Viviennne Amanda Curtis; Margaret Susannah D'Ambrogio; Tamal De; Corinne Genevieve de Sousa; Roger Stephen Heaton Denny; Ian George Dewar; M S Dharmendra; Deepa Dighe-Deo; Jane Ann Dixon; Gerald Patrick Michael Doyle; Marguerite Anne Doyle; Flonnuala Mary Dunne; Mark John Alan Earthrowl; Ahmed Mohamed Refaat Ahmed El-Dosoky: Nakanalini Elanchenny; Melanie Evans; Paul Francis Fearon; Lakshmi Fernandes; Brian Fitzmaurice; Samuel William Forshall: Flonuala Delia Maria Galvin; Mohan George; Carmel Philomena Gibbons; Simon Martin Gilbody: Catherine Anne Gordon; Clifford John Haley; Mary Anne Harty; Margaret Mary Heap; Emma Louise Heap; Johnanna Jane Herrod; Stephen Andrew John Hinder; Caroline Lesley Holmes; Sarah Jane Huline-Dickens; Alastair Marcus Hull; Mark Antoni Janas; Akdla Indrajith Jayasekera; Praveen Jetty; Ian Richard Jones; Russell Hay Keen; Simon James Hamilton Kelly: Shakdl Salam Khawaja; Christopher George Krasuckd; Shailesh Kumar; Denise Ellen Kyte; Catherine Lavelle; Tak Shing Lee; Ravi Yagasekaran Lingam; Beverley Elizabeth Louis: Gail Elizabeth Loveday: Thomas Hood MacEwan; Therese Nimal Markar; Jane Christine Martin; Angela Pauline McAleer; Anne Patricia McDonnell; Stephen William McGowan; Mairi Ruth McGowan; Nirmalie Mirando; John Farnill Morgan; Julian Michael Bedford Morrell; Faiza Hassan Muft;: Alison Clare Munden; Alan William O'Donohoe; Donal Thomas John O'Hanlon; Chinyere Constance Ojiegbe; Anthony Bassey Okore; Anne Catherine Perry; Gillian Tracy Pinner; Richard John Porter; Jonathan Raymond Price; Priscilla Elise Read; Harvey James Rees; Christopher Mark Roberts; Ian Michael Rogerson; Khalid Saeed; Swaroopa Saha; Aunmita Saha; Angel Santos Ramon; Rhona Jean Sargeant; Kathri Achchige Sandya Satarasinghe; Lucinda Valerie Scott; Alice Ellen Seabourne; Ptyal Sen; Jordi Serra Mestres; Sukhwinder Singh Shergill; Annie Kamala Simon; Anushtayini Stvananthan; Shubulade Mary Eniola Smith; Craig Anthony Smith; Daniel Kenneth Smith; Melanie Jane Sore; Greg Spencer; Catherine Jayne Staff; Gertl Stegen; John Patrick Stephens; Thomas Guy Stevens; Flona Claire Stormont; Joanne Shirley Stubley;
Athula Sumathipala; Annette Thampi; Molliamma Thomas; Judy Heng Yuen Too; Steve Tso; Mark Andrew Turner; Guy Marcus Undrill; Elizabeth Judith Vanhorn; John Vickers; Fiona Marie Vickers; Catherine Louise Wainhouse; Anne Marie Waldron: Michael Henry Walker; Nicholas Paul Walker; David Anthony Ward; David Andrew Watson; Kurt Jon Wendelborn; Andrew Fleming West; Mary-Ellen White; Helen Elizabeth Whitworth; Simon William Wilson; Nicole Winter; Grace Josephine Wing San Wong; Ian Cosmo Wright; Nwe Nwe Wynn.

\section{Laughlin Prize}

The Laughlin Prize has been awarded jointly to $\mathrm{Dr}$ Mary Nicola Clarke and Dr Melanie Evans.

The College would like to thank the following hospitals for hosting the Autumn 1995 Examinations. Special thanks to the Senior Organisers, the hospital staff and the patients involved.

\section{Part I}

Dr P. McGarry

Dr B. Robinson

Dr C. A. Lawton

Dr R. N. Chitty

Dr M. J. Smith

Knockbracken

Healthcare Park, Belfast

Barrow Hospital, Bristol Addenbrooke's Hospital, Cambridge

W Cheshire NHS Trust. Chester North Tees General Hospital, Cleveland

Dr C. B. Sarathchandra Colchester General

Dr A. Beveridge Hospital, Colchester Queen Margaret Hospital, Dunfermline

Dr P. Jauhar

Dr C. T. Methven

Professor M. Reveley Parkhead Hospital, Glasgow Denmark Road Day Hospital, Gloucester Leicester General Hospital, Leicester

Dr A. Puranik

Dr K. Richard

Dr P. A. Childs

Dr J. M. Annear Medway Hospital, Medway

Murray Royal Hospital, Perth

Royal South Hants

Hospital, Southampton

Warlingham Park

Hospital, Surrey

\section{Part II}

Dr J. S. Callender
Royal Cornhill Hospital, Aberdeen 
Dr G. Henry

Dr A. P. Roberts

Dr L. B. Campbell

Professor T. J. Fahy

Dr A. F. Cooper

Dr R. W. Latcham

Dr M. Courtney

Dr V. Sharma
Holywell Hospital, Antrim Hergest Unit, District General Hospital,

Bangor

Bethlem Royal Hospital,

Kent

Untversity College

Hospital, Galway

Leverndale Hospital,

Glasgow

Hinchingbrooke Health

Care NHS Trust,

Huntingdon

St James's University

Hospital, Leeds

Fazakerley Hospital, Liverpool
Dr M. George

Dr S. Fleminger

Dr M. Pierides

Dr Gabriella Zolese

Dr Ian Collis

Dr L. D. Mulgirigama

Dr D. Battin
Queen Elizabeth Psychiatric Hospital, Birmingham

The Royal London

Hospital, London

South Western

Hospital, Royal London

Springfield Hospital, London

St Luke's Woodside

Hospital, London

Royal Earlswood

Hospital, Surrey

Worcester Royal

Infirmary, Worcester

\section{Miscellany}

\section{Professor Tom Arie}

Professor Tom Arie has been awarded the British Geriatrics Society's Dhole-Eddleston Memorial Prize for outstanding contributions to the literature on the medical care of older people.

\section{New publications}

The African Journal of Psychiatry is due to be back in circulation after a break of nearly fifteen years. While the Journal style of the previous series will be largely retained, some modifications will be necessary in order to bring the new series up to contemporary standards. Further information or those wishing to submit articles for publication, books for review or subscriptions should contact Professor Olufemi Morakinyo, Editor in Chief, African Journal of Psychiatry, c/o Department of Mental Health, Obafemi Awolowo University, Ile-Ife, Nigeria.

Assessment of Mental Capacity: Guidance for Doctors and Lawyers-A joint report of the BMA and the Law Society, has been published by the British Medical Association and is available at
\&8.95 from the BMA and the Law Society Shop (Telephone: 0171242 1222).

Making Sense of Treatments and Drugs: ECT, has been produced by MIND in an attempt to improve the information available to patients about ECT treatment. This is available from MIND Publications for £2.50. Further information: MIND, 1519 Broadway, Stratford, London E15 4BQ.

The World Health Organization has produced a set of Behavioural Science Learning Modules in order to provide Schools of Medicine and others within the health area with materials to improve the teaching of issues related to health and behaviour. Recent modules aimed specifically at communication aspects of health care are Communicating Bad News (to patients), Introducing Parents to their Abnormal Baby, and Improving Adherence Behaviour with Treatment Regimens. Other modules are available, focusing on clinical material. Further information: WHO Programme on Mental Health, 1211 Geneva 27, Switzerland (Fax: ++41 22791 0746). 